\title{
Clinical Features of Kawasaki Disease during SARS- CoV-2 Epidemic: A Single-Center Retrospective Study
}

\author{
Toshimasa Nakada
}

\section{ABSTRACT}

\begin{abstract}
The severe acute respiratory syndrome coronavirus 2 (SARS-CoV-2) epidemic, causing coronavirus disease 2019 (COVID-19), has rapidly spread worldwide. Recently, cases of Kawasaki-like syndrome associated with COVID-19 (MIS-C/PIMS) have been reported in the USA and Europe. However, the clinical features of Kawasaki disease (KD) in Japan during the SARS-CoV-2 epidemic remain unclear. Therefore, this retrospective study aimed to evaluate the clinical features of $\mathrm{KD}$ in our department during the year of the COVID-19 pandemic (2020). Data were obtained from $45 \mathrm{KD}$ patients who underwent acute-phase treatment from January 2018 to December 2020. The patients were divided into three groups: (1) 2018 group, in which 18 patients receiving acute-phase treatment during 2018; (2) 2019 group, in which 17 patients receiving acute-phase treatment during 2019; and (3) 2020 group, in which 10 patients receiving acute-phase treatment during 2020 . The comparison of the clinical findings, treatments, and outcomes among the three groups (2018 vs. 2019 vs. 2020 group) revealed a significant difference in the rate of the male gender $(72.2 \%$ vs. $41.2 \%$ vs. $10.0 \%, P<0.010)$, recurrence $(0.0 \%$ vs. $0.0 \%$ vs. $25.0 \%, P=0.015)$, and coronary artery lesions $(16.7 \%$ vs. $0.0 \%$ vs. $0.0 \%, P=0.044)$. No patients required ventilation assistance or inotropic agents and met the diagnostic criteria of MIS-C/PIMS. Further studies are warranted to elucidate the clinical features of KD during the SARS-CoV-2 epidemic.
\end{abstract}

Keywords: COVID-19, Kawasaki disease, male, recurrence, SARS-CoV-2.
Published Online: June 15, 2021

ISSN: $2736-5476$

DOI: $10.24018 /$ ejclinicmed.2021.2.3.33

\section{T. Nakada*}

Department of Pediatrics, Aomori Prefectural Central Hospital, Aomori City, Japan.

(e-mail: toshimasanakada ${ }^{\circledR}$ yahoo.co.jp)

*Corresponding Author

\section{INTRODUCTION}

Kawasaki disease (KD) is a form of acute febrile systemic vasculitis that primarily affects children below 5 years old [1]. Coronary artery lesions (CALs) are a severe complication of KD [2].

The severe acute respiratory syndrome coronavirus 2 (SARS-CoV-2) epidemic, causing coronavirus disease 2019 (COVID-19), has rapidly spread worldwide [3]. Recently, cases of Kawasaki-like syndrome associated with COVID-19 have been reported in the USA and Europe [3]-[6]. The Centers for Disease Control and Prevention and the European Centre for Disease Prevention and Control issued alerts concerning Kawasaki-like syndrome associated with COVID-19. This syndrome is also known as multisystem inflammatory syndrome in children (MIS-C) and pediatric inflammatory multisystem syndrome (PIMS) [7], [8]. To date, no reports of this syndrome from East Asia have been observed despite the high prevalence of KD in the area [9].

No dramatic increase in KD incidence or changes in its clinical features were observed during the local COVID-19 epidemic in Japan, unlike in other countries where MISC/PIMS is endemic [10]. A study that adopted meta-analysis suggests that MIS-C/PIMS leads to multiple organ failure, including gastrointestinal manifestations, myocardial dysfunction, and coronary abnormalities, and has distinct features from KD [11]. However, the clinical features of KD in Japan during SARS-CoV-2 epidemic remain unclear. Therefore, this retrospective study aimed to evaluate the clinical features of $\mathrm{KD}$ in our department during the year of COVID-19 pandemic.

\section{Participants AND Methods}

The study protocol was approved by our institutional ethics committee, and the requirement of patient consent was waived owing to the retrospective nature of the study.

Data were obtained from $45 \mathrm{KD}$ patients who underwent acute-phase treatment in our department from January 2018 to December 2020. The patients who received acute-phase treatment were divided into three groups: (1) 2018 group, in which 18 patients during 2018; (2) 2019 group, in which 17 patients during 2019; and (3) 2020 group, in which 10 patients during 2020 .

KD was diagnosed based on the criteria (Japanese, fifth edition) mentioned in the diagnostic guidelines for KD until August 2019 and on the revised criteria (Japanese, sixth edition) from September 2019 [2], [12]. Intravenous immunoglobulin (IVIG) resistance was defined as a fever that persisted or reappeared $24 \mathrm{~h}$ after the first course of treatment [13]. Recurrent KD was defined as a secondary presentation of another episode 2 months following the initial episode [14], [15]. The Egami score, a risk score for predicting IVIG 
resistance based on clinical findings, including age, days of illness, platelet count, alanine aminotransferase level, and Creactive protein level, was evaluated before the initial IVIG therapy [16].

\section{A. Initial Therapy}

During the study period, a single IVIG infusion at 2 $\mathrm{g} / \mathrm{kg} / \mathrm{dose}$ was administered as initial therapy starting on day 5 of the illness, whenever possible [17]. Patients without inflammation or complications upon presentation underwent acute-phase therapy without IVIG [17]. During the study period, low-dose aspirin ( $5 \mathrm{mg} / \mathrm{kg} /$ day) was started on days $8-10$ of the illness following the completion of IVIG infusion, including the second course of the therapy [17]. Mediumdose aspirin (30 mg/kg/day) was started if the patients had $\mathrm{CAL}$ or were candidates for plasma exchange.

\section{B. Rescue Therapy}

The decision to use rescue therapies in resistant patients was made 48 to $72 \mathrm{~h}$ following the completion of the initial IVIG infusion. Physicians made this decision using comprehensive clinical parameters, including body temperature, major KD symptoms, general condition, and laboratory data [17]. Course 2 of the therapy comprised rescue IVIG infusion at $2 \mathrm{~g} / \mathrm{kg} / \mathrm{dose}$, and course 3 comprised ulinastatin infusion, third course of IVIG therapy, or plasma exchange [17].

\section{Diagnosis of CAL}

CALs were diagnosed via echocardiography based on the Japanese criteria reported by Kobayashi et al [18]. CAL was diagnosed if any examination revealed an internal lumen diameter of $\geq 3 \mathrm{~mm}$ in a patient younger than 5 years or a diameter of $\geq 4 \mathrm{~mm}$ in a patient older than 5 years if the internal diameter of a segment was at least 1.5 times larger than that of an adjacent segment or if the lumen appeared irregular. A transient CAL was defined as the disappearance of a CAL within 30 days of the illness.

\section{Statistical Analysis}

Statistical analyses were conducted using Stat Flex Version 6 for Windows (Artech Co., Ltd., Osaka, Japan). Chisquare test, Fisher exact test, Mann-Whitney U test, and Kruskal-Wallis test were used accordingly, with sample size considerations. $\quad \mathrm{P}<0.05$ was considered statistically significant.

\section{RESULTS}

The numbers of the patients in the 2018, 2019, and 2020 groups were 18, 17, and 10, respectively (Fig. 1 and Table I). The rate of the number of the patients in 2020 group was $58.8 \%$ of that of the 2019 group (Fig. 1). Out of 10 patients, 2 in the 2020 group had both the initial and the second presentation (recurrence) of KD in the same year. One patient who had the initial presentation of KD in 2015 had the second presentation (recurrence) in 2020. Therefore, a total of 10 patients had 12 presentations of KD in 2020 (Table I). Three presentations of KD were recurrences in the 2020 group (Table I), and those three patients with KD recurrence were of female sex.

Two patients in 2020 group underwent polymerase chain reaction test for SARS-CoV-2, and they had negative results. No patients required ventilation assistance or inotropic agents and met the diagnostic criteria of MIS-C/PIMS [4], [19].

The comparison of clinical findings, treatments, and outcomes among the three groups revealed a significant difference in the rate of the male gender, recurrence, and CAL (Table I). One patient in the 2018 group had medium sized CALs before initial therapy.

\section{DISCUSSION}

The main findings of this study were the reduced number of KD patients in the 2020 group and the significant difference in the rate of the male gender, recurrence, and CAL among the three groups (Fig. 1, Table I).

One study revealed that the COVID-19 pandemic dramatically reduced admissions of children with and without chronic conditions to general pediatric wards [20]. The number of daily hospitalizations declined during the COVID19 period compared with that of the immediate period before COVID-19 and that of 2018 and 2019 [20]. This indicated a reduction of 59\% [20]. Furthermore, a questionnaire survey conducted by Japanese Society of KD in 2020 indicated a reduction of about $50 \%$ admissions of children with KD [21]. Those data were consistent with the results of the present study (Fig. 1).

A possible contributing factor to the decrease in the number of children with KD may be the reduction in the transmission of infectious disease due to COVID-19 restrictions [20]. A significant decrease in hospitalizations due to viral and bacterial infections during the COVID-19 period was observed [20]. The epidemiological findings indicate that $\mathrm{KD}$ is mediated by several infectious agents, which triggers its onset [22], [23]. The decrease in the transmission of infectious disease may lead to the decrease in the incidence of KD. In fact, the number of the patients with infectious disease who required admission was decreased in our catchment area in the year of COVID-19 pandemic as well as the number of those with KD.

Parents may have been scared of getting infected with COVID-19 and avoided going to hospital [20]. Therefore, some patients with KD with mild severity might not consult a doctor. This may be another factor of the decreased number of patients with KD during the COVID-19 pandemic. The impact of delaying treatment may take weeks, months, or years to become obvious, especially if children have a risk of developing CAL. Fortunately, patients with the delayed acute-phase treatment and with CAL caused by KD at present have not been experienced. One study regarding adult acute coronary syndrome revealed the increased proportion of delayed admissions and the increase in the severity of patients with the disease during the COVID-19 pandemic in Japan compared with those observed during previous years [24].

No patients in the present study met the diagnostic criteria of MIS-C/PIMS. Most cases of MIS-C and PIMS following COVID-19 were reported in the USA and Europe, with no cases thus far being reported in East Asia, including China, South Korea, Taiwan, and Japan [9]. Recent report from Japan revealed that small number of children had severe COVID-19 [25]. Furthermore, the pooled proportions of Hispanic and Black cases were $34.6 \%$ and $31.5 \%$, 
respectively [11]. These facts led to the speculation with regard to the variations in genomic susceptibility [9], [10]. Fewer children having COVID-19 may be another reason for no reports of MIS-C/ PIMS cases in Japan [10].

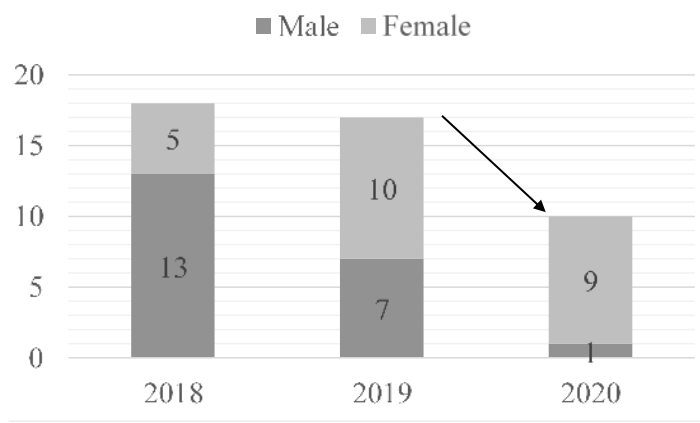

Fig. 1. The numbers and gender of the patients with Kawasali disease in 2019, 2019 an 2020 groups.

TABLE I: COMPARISON OF CLINICAL FINDINGS, TREATMENTS AND OUTCOMES AMONG THREE GROUPS

\begin{tabular}{|c|c|c|c|c|}
\hline Variables & $\begin{array}{l}2018 \text { group } \\
(\mathrm{n}=18)\end{array}$ & $\begin{array}{l}2019 \text { group } \\
(\mathrm{n}=17)\end{array}$ & $\begin{array}{l}2020 \text { group } \\
(\mathrm{n}=12)\end{array}$ & P-value \\
\hline Male gender & $13(72.2 \%)$ & $7(41.2 \%)$ & $\begin{array}{c}1(10.0 \%) \\
(\mathrm{n}=10)\end{array}$ & $<0.010$ \\
\hline $\begin{array}{c}\text { Age at KD } \\
\text { onset (months) }\end{array}$ & $\begin{array}{c}29.5(18.0- \\
45.0)\end{array}$ & $\begin{array}{c}28.0(15.3- \\
44.3)\end{array}$ & $\begin{array}{c}24.5(20.5- \\
40.0)\end{array}$ & 0.857 \\
\hline $\begin{array}{c}\text { Incomplete } \\
\text { type }\end{array}$ & $4(22.2 \%)$ & $3(17.6 \%)$ & $1(8.3 \%)$ & 0.342 \\
\hline Egami score & $1.5(1.0-3.0)$ & $1.0(0.0-2.0)$ & $1.0(0.0-1.5)$ & 0.110 \\
\hline Recurrence & $0(0.0 \%)$ & $0(0.0 \%)$ & $3(25.0 \%)$ & 0.015 \\
\hline IVIG therapy & $17(94.4 \%)$ & $15(88.2 \%)$ & $12(100.0 \%)$ & 0.693 \\
\hline $\begin{array}{l}\text { Start day of } \\
\text { illness of } \\
\text { initial IVIG } \\
\text { therapy }\end{array}$ & $5.0(5.0-6.0)$ & $5.0(5.0-6.0)$ & $5.5(5.0-6.0)$ & 0.820 \\
\hline Non-responder & $\begin{array}{c}5(29.4 \%) \\
(\mathrm{n}=17)\end{array}$ & $\begin{array}{c}4(26.7 \%) \\
(n=15)\end{array}$ & $\begin{array}{c}5(41.7 \%) \\
(\mathrm{n}=12)\end{array}$ & 0.664 \\
\hline $\begin{array}{l}\text { Rescue } \\
\text { therapy } \\
\text { for non- } \\
\text { responder } \\
\text { Aspirin }\end{array}$ & $\begin{array}{c}2(11.8 \%) \\
(\mathrm{n}=17)\end{array}$ & $\begin{array}{c}2(13.3 \%) \\
(\mathrm{n}=15)\end{array}$ & $\begin{array}{c}4(33.3 \%) \\
(\mathrm{n}=12)\end{array}$ & 0.174 \\
\hline Low-dose & $16(88.9 \%)$ & $17(100.0 \%)$ & 11 (91.7\%) & 0.594 \\
\hline $\begin{array}{l}\text { Medium-dose } \\
\text { CAL }\end{array}$ & $2(11.1 \%)$ & $0(0.0 \%)$ & $1(8.3 \%)$ & \\
\hline $\begin{array}{l}\text { Less than one } \\
\text { month after } \\
\text { KD onset }\end{array}$ & $3(16.7 \%)$ & $0(0.0 \%)$ & $0(0.0 \%)$ & 0.044 \\
\hline $\begin{array}{c}\text { One month } \\
\text { after KD onset }\end{array}$ & $2(11.1 \%)$ & $0(0.0 \%)$ & $0(0.0 \%)$ & 0.103 \\
\hline
\end{tabular}

Data are presented as $\mathrm{n}(\%)$ or median (interquartile range).

KD: Kawasaki disease, IVIG: intravenous immunoglobulin, CAL: coronary artery lesion.

The decreased number of the patients with $\mathrm{KD}$ and the lower ratio of male to female may be due to bias based on the small sample size of this study. A higher male-to-female ratio exists for KD [26]. One epidemiological study in Japan revealed that the male-to-female ratio of patients with $\mathrm{KD}$ between 2015 and 2016 was 1.34 [23].

In one study, data for 330,662 patients reported in $\mathrm{KD}$ nationwide surveys in Japan implemented between 1961 and 2014 were chronologically compared based on patient age distribution, estimated mean age of onset, and male-to-female ratio during four periods: pre-epidemic (P1), epidemic (P2), stable (P3), and recent (P4): 1961-1978, 1979-1986, 19871997, and 1998-2014, respectively [22]. The conclusion of this study indicated that P2 and P4 were significantly different in epidemiological features and that the male-tofemale ratio tended to decrease during P2 [22]. The male-tofemale ratio tended to decrease with the increasing number of patients with KD in Japan [22]. The low male-to-female ratio in increased patients with KD with the influenza $\mathrm{A}(\mathrm{H} 1 \mathrm{~N} 1)$ pandemic in France was also reported [6]. Inversely, the decreased male-to-female ratio was observed despite the decreased number of patients with KD in the year of COVID19 pandemic (2020) in the present study (Fig. 1, Table I).

One epidemiological study revealed that the incidence of recurrent $\mathrm{KD}$ has remained largely unchanged over the past 30 years, and risk factors for KD recurrence included male sex [27]. In the present study, the rate of recurrent KD of the 2020 group was significantly high among the three groups, and three patients with KD recurrence were of female sex. The results regarding KD recurrence and gender in the present study were different from those of the previous epidemiological study [6], [22], [27]. Further studies are warranted to elucidate the clinical features of $\mathrm{KD}$ during SARS-CoV-2 epidemic.

One patient in the 2018 group had medium-sized CALs before the initial therapy. A study revealed that all patients with persistent CALs 1 year after KD onset already had an abnormal initial echocardiogram and that the presence of any abnormalities on the initial echocardiogram may contribute to the early identification of patients with severe disease [28]. Another study using multivariable analyses demonstrated that the lack of CAL regression and persistent large CAL in the late-phase were associated with large coronary artery diameters at diagnosis [29]. The study using logistic regression analysis of our patients with $\mathrm{KD}$ also indicated that CAL before the initial treatment is an independent risk factor for persistent CAL in late-stage $\mathrm{KD}$ [30]. Therefore, one of the reasons for the higher rate of CALs in the 2018 group may be the presence of the patient with CALs before the initial therapy.

This study has two limitations, namely, the small sample size and the retrospective study design.

In conclusion, the reduced number of patients with $\mathrm{KD}$ who underwent acute-phase treatment, the lower male-tofemale ratio, and higher KD recurrence rate in our department were observed during the SARS-CoV-2 epidemic in 2020. Further studies are warranted to elucidate the clinical features of KD during the COVID-19 pandemic.

\section{ACKNOWLEDGMENT}

I would like to thank all of those who were involved in the medical management of the patients included in this study, and Enago (www.enago.jp) for the English language review.

\section{REFERENCES}

[1] Kawasaki T, Kosaki F, Okawa S, Shigematsu I, Yanagawa H. A new infantile acute febrile mucocutaneous lymph node syndrome (MLNS) prevailing in Japan. Pediatrics 1974; 54: 271-6.

[2] Fukazawa R, Kobayashi J, Ayusawa M, Hamada H, Miura M, Mitani Y, et al. JCS/JSCS 2020 Guideline on Diagnosis and Management of Cardiovascular Sequelae in Kawasaki Disease. Circ J 2020; 84: 1348407. doi:10.1253/circj.CJ-19-1094. 
[3] Verdoni L, Mazza A, Gervasoni A, Martelli L, Ruggeri M, Ciuffreda $\mathrm{M}$, et al. An outbreak of severe Kawasaki-like disease at the Italian epicentre of the SARS-CoV-2 epidemic: an observational cohort study. Lancet 2020; 395: 1771-78. https://doi.org/10.1016/S01406736(20)31103-X.

[4] LR Feldstein, EB Rose, SM Horwitz, JP Collins, MM Newhams, MBF Son, et al. Multisystem Inflammatory Syndrome in U.S. Children and Adolescents. N. Engl. J. Med. 2020; 383: 334-46. DOI: 10.1056/NEJMoa2021680.

[5] Whittaker E, Bamford A, Kenny J, Kaforou M, Jones CE, Shah P, et al. Clinical characteristics of 58 children with a pediatric inflammatory multisystem syndrome temporally associated with SARS-CoV-2. JAMA. 2020;324(3):259. doi:10.1001/jama.2020.10369.

[6] Ouldali N, Pouletty M, Mariani P, Beyler C, Blachier A, Bonacorsi S, et al. Emergence of Kawasaki disease related to SARS-CoV-2 infection in an epicentre of the French COVID-19 epidemic: a time-series analysis. Lancet Child Adolesc Health 2020; 4: 662-68. https://doi.org/10.1016/S2352-4642(20)30175-9.

[7] The Centers for Disease Control and Prevention. Multisystem Inflammatory Syndrome in Children (MIS-C) Associated with Coronavirus Disease 2019 (COVID-19). https://emergenc y.cdc.gov/han/2020/han00432.asp. (Accessed on July 19, 2020).

[8] Royal College of Paediatrics and Child Health. Guidance-Paediatric multisystem inflammatory syndrome temporally associated with COVID-19. https://www.rcpch.ac.uk/sites/default/files/202005/COVID-19-Paediatric-

ultisystem-\%20inflammatory\%20syndrome-20200501.pdf. (Accessed on July 19,2020).

[9] Uda K, Okita K, Soneda K, Taniguchi K, Horikoshi Y. Kawasaki disease following coronavirus disease 2019 with prolonged fecal viral shedding. Pediatr Int (2020) 0, 1-3 doi: 10.1111/ped.14452.

[10] Iio K, Uda K, Hataya H, Yasui F, Honda T, Sanada T, et al. Kawasaki disease or Kawasaki-like disease: Influence of SARSCoV-2 infections in Japan. Acta Paediatrica. 2021; 110:600-1. DOI: 10.1111/apa.15535.

[11] Yasuhara J, Watanabe K, Takagi H, Sumitomo N, Kuno T. COVID-19 and multisystem inflammatory syndrome in children: A systematic review and meta-analysis. Pediatric Pulmonology. 2020;1-12. DOI: $10.1002 /$ ppul.25245.

[12] Ayusawa M, Sonobe T, Uemura S, Ogawa S, Nakamura Y, Kiyosawa $\mathrm{N}$, et al. Revision of diagnostic guidelines for Kawasaki disease (the 5th revised edition). Pediatr Int 2005; 47: 232-4 https://doi.org/10.1111/j.1442-200x.2005.02033.

[13] Rearch Committee of the Japanese Society of Pediatric Cardiology; Cardiac Surgery Committee for Development of Guidelines for Medical Treatment of Acute Kawasaki Disease. Guidelines for medical treatment of acute Kawasaki disease: report of the Research Committee of the Japanese Society of Pediatric Cardiology and Cardiac Surgery (2012 revised version). Pediatr Int 2014; 56: 135-58. https://doi.org/10.1111/ped.12317.

[14] Hirata S, Nakamura Y, Yanagawa H. Incidence rate of recurrent Kawasaki disease and related risk factors: from the results of nationwide surveys of Kawasaki disease in Japan. Acta Paediatr. 2001; 90(1): 40-4. https://doi.org/10.1111/j.1651-2227.2001.tb00253.x.

[15] Nakada T. Usefulness of an initial single intravenous immunoglobulin infusion with delayed use of aspirin against Kawasaki disease relapse: a single-center retrospective study. Journal of Advanced Research in Medicine 2019; 6 (1): 18-24. https://doi.org/10.24321/2349.7181.201905.

[16] Egami K, Muta H, Ishii M, Suda K, Sugahara Y, Iemura M, et al. Prediction of resistance to intravenous immunoglobulin treatment in patients with Kawasaki disease, J Pediatr 2006; 149: 237-40.

[17] Nakada T. Acute Phase Treatment and Medium-Term Outcomes in Kawasaki Disease. European Journal of Medical and Health Sciences 2020; 2 (4), August: 1-7. http://dx.doi.org/10.24018/ejmed.2020.2.4.419.

[18] Kobayashi T, Inoue Y, Takeuchi K, OkadaY, Tamura K, Tomomasa T, et al. Prediction of intravenous immunoglobulin unresponsiveness in patients with Kawasaki disease. Circulation 2006; 113: 2606-12. http://www.circulationaha.org.DOI:10.1161/CIRCULATIONAHA.10 5.592865 .

[19] World Health Organization. Multisystem inflammatory syndrome in children and adolescents with COVID-19. Published May 15, 2020. Accessed May 22, 2020. https://www.who. int/publicationsdetail/multisystem-inflammatorysyndrome-in-children-andadolescents-withcovid-19.

[20] Gavish R, Levinsky Y, Dizitzer Y, Bilavsky E, Livni G, Pirogovsky A, et al. The COVID-19 pandemic dramatically reduced admissions of children with and without chronic conditions to general paediatric wards. Acta Paediatrica 2021; 00: 1-6. DOI: 10.1111/apa.15792.
[21] Homepage of Japanese Society of Kawasaki disease. 8 January 2021 http://www.jskd.jp/pdf/KD-COVID-Questionnaire0108.pdf.

[22] Tomita Y, Shimaya M, Yamaura Y, Tsujiguchi R, Takahashi K, Fukaya T. Kawasaki disease: Epidemiological differences between past and recent periods, and implications of distribution dynamism. Pediatr Int. 2018; 60(4): 349-56. doi: 10.1111/ped.13522.

[23] Makino N, Nakamura Y, Yashiro M, Kosami K, Matsubara Y, Ae R, et al. Nationwide epidemiologic survey of Kawasaki disease in Japan, 2015-2016. Pediatr Int 2019; 61: 397-403. https://doi.org/10.1111/ped.13809.

[24] Yasuda Y, Ishiguchi H, Ishikura M, Yoshida M, Imoto K, Sonoyama $\mathrm{K}$, et al. Incidence and Demographic Trends for Acute Coronary Syndrome in a Non-Epidemic Area During the Coronavirus Disease Pandemic in Japan - A 2-Institutional Observational Study - Circ Rep 2021; 3: 95-9. doi:10.1253/circrep.CR-20-0141.

[25] Homepage of Japanese Society of Kawasaki disease. 25 February 2021. http://www.jskd.jp/pdf/210225MIS-C.pdf.

[26] Cattalini M, Soliani M, Costanza Caparello MC, Cimaz R. Sex Differences in Pediatric Rheumatology. Review Clin Rev Allergy Immunol. 2019; 56(3): 293-307. doi: 10.1007/s12016-017-8642-3.

[27] Sudo D, Nakamura Y. Nationwide surveys show that the incidence of recurrent Kawasaki disease in Japan has hardly changed over the last 30 years. Acta Paediatrica 2017; 106 (5): 796-800. https://doi.org/10.1111/apa.13773.

[28] Chbeir D, Gaschignard J, Bonnefoy R, Beyler C, Melki I, Faye A, et al Kawasaki disease: abnormal initial echocardiogram is associated with resistance to IVIg and development of coronary artery lesions. Pediatr Rheumatol Online J. 2018; 16(1): 48. https://doi.org/10.1186/s12969018-0264-7.

[29] Friedman KG, Gauvreau K, Hamaoka-Okamoto A, Tang A, Berry MS, Tremoulet A, et al. Coronary artery aneurysms in Kawasaki disease: risk factors for progressive disease and adverse cardiac events in the US population. J Am Heart Assoc. 2016; 5: e003289. https://doi:10.1161/JAHA.116.003289.

[30] Nakada T. Outcomes in patients with Kawasaki disease associated with coronary artery lesions before initial therapy. Med J Aomori 2020; 65 (1): $1-7$.

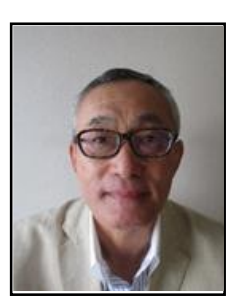

Dr. T. Nakada, Birth of place: Japan. Birth of date: $20 / 02 / 1957$.

$\mathrm{He}$ received medical education at Hirosaki University School of Medicine. He had MD regarding pediatrics. He has been working as a pediatrician at Department of Pediatrics, Aomori Prefectural Central Hospital. Aomori City, Aomori Prefecture, Japan, from 1988 to the present time.

His major field of study is general pediatric cardiology: Kawasaki disease, arrhythmia, congenital heart disease, cardiomyopathy, heart disease screening, and preventive medicine. Acute phase treatment for Kawasaki disease using an initial single intravenous immunoglobulin therapy is one of his lifeworks. 